\title{
Quantum measurement and feedback control
}

\author{
Luc Bouten, Ramon van Handel, Anthony Miller, Gopal Sarma, and Hideo Mabuchi \\ California Institute of Technology 266-33, Pasadena, CA 91125 USA
}

Real-time signal processing and feedback control are key aspects of classical engineering. As we progress into a new era of quantum engineering, it stands to reason that improved understandings of quantum continuous measurement and real-time feedback could provide similarly empowering methodology. Quantum optics is in many ways an ideal physical setting in which to begin to explore such topics, as there is excellent contact between experiment and ab initio theoretical modeling. What is needed now are case studies in applying the central ideas of real-time filtering and feedback control to problems of practical technological and/or scientific interest, and compelling demonstrations of the novel capabilities such an approach can bring.

One can already identify several interesting themes in the early development of quantum control theory: the application of "off-the-shelf" technical tools from classical control engineering to develop improved strategies in scenarios involving quantum dynamics and noise; the adaptation of classical feedback control theory to incorporate modern formulations of quantum measurement theory, and practical applications of the resulting methods; and preliminary investigations of the novel topic of coherent quantum feedback control. Examples of work in the first theme include numerous applications of semi-definite programming and relaxation methods in quantum information theory. Examples of the second include applications of feedback control in the development of new receiver designs for quantum-optical telecom. The third theme is just beginning to emerge as an active research area, linking fundamental issues in quantum field theory with new challenges for applied and computational mathematics in non-commutative stochastic analysis and optimization.

Our aim in this invited presentation will be to give a flavor of each of these three themes via an overview of present-day research in quantum control. Much of the discussion will focus on theoretical investigations of models that are close to experiment, but we will make connections to real laboratory efforts and results where possible. 